\title{
On the age of the TW Hydrae association and $2 \mathrm{M} 1207334-393254^{\star, \star \star}$
}

\author{
D. Barrado y Navascués

\begin{abstract}
Laboratorio de Astrofísica Espacial y Física Fundamental, LAEFF - INTA, PO Box 50727, 28080 Madrid, Spain e-mail: barrado@laeff.inta.es
\end{abstract}

Received 29 May 2006 / Accepted 4 August 2006

\begin{abstract}
Aims. We have estimated the age of the young moving group TW Hydrae Association, a cohort of a few dozen stars and brown dwarfs located near the Sun which share the same kinematic properties and, presumably, the same origin and age.

Methods. The chronology has been determined by analyzing different properties (magnitudes, colors, activity, lithium) of its members and comparing them with several well-known star forming regions and open clusters, as well as theoretical models. In addition, by using medium-resolution optical spectra of two M8 members of the association (2M1139 and 2M1207 - an accreting brown dwarf with a planetary mass companion), we have derived spectral types and measured $\mathrm{H} \alpha$ and lithium equivalent widths. We have also estimated their effective temperature and gravity, which were used to produce an independent age estimation for these two brown dwarfs. We have also collected spectra of 2M1315, a candidate member with a L5 spectral type and measured its H $\alpha$ equivalent width.

Results. Our age estimate for the association, $10_{-7}^{+10} \mathrm{Myr}$, agrees with previous values cited in the literature. In the case of the two brown dwarfs, we have derived an age of $15_{-10}^{+15} \mathrm{Myr}$, which also agree with our estimate for the whole group.

Conclusions. We compared our results with recent articles published on the same subject using other techniques, and discuss the limits of the age-dating techniques.
\end{abstract}

Key words. Galaxy: open clusters and associations: individual: TW Hydrae Association - stars: low-mass, brown dwarfs stars: pre-main-sequence - stars: planetary systems - Galaxy: open clusters and associations: general - stars: general

\section{Introduction}

If we want to put any astronomical phenomenon in context, we need to know its evolutionary status, i.e. its age. This is even more important when the phenomenon is a benchmark in a given discipline. This is the case of the TW Hydrae Association or TWA (de la Reza et al. 1989; Kastner et al. 1997), a young, nearby moving group which includes a few dozen stars and brown dwarfs (see the recent review by Zuckerman \& Song 2004). The age has been estimated in the range 8-20 Myr (Kastner et al. 1997; Stauffer et al. 1995; Soderblom et al. 1998; Hoff et al. 1998; Weintraub et al. 2000; Makarov \& Fabricius 2001; Makarov et al. 2005; de la Reza et al. 2006), and the individual distances, from Hipparcos, are in the range 47-67 pc (ESA 1987). Due to these characteristics, the TWA is becoming a reference for such phenomena as accretion and circumstellar disks, rotation and activity, the formation of planetary systems and the direct detection of planets.

Regarding the latter issue, members of the association have been the targets of several searches for planetary companions, using AO techniques. Of those studies which have been published, the most comprehensive one has been that by Masciadri et al. (2004), who observed 30 targets with VLT/NACO, including six stellar members of TWA, with no positive result.

\footnotetext{
* Based on observations collected with the Magellan Telescopes.

$\star \star$ Table 1 and Figs. 2, 4, 7-9, 13 are only available in electronic form at http://www . aanda.org
}

However, Chauvin et al. (2004) announced the discovery of a candidate companion of the brown dwarf 2M1207334-393254 (2M1207 hereafter), which has a mass estimate within the planetary domain $\left(5 M_{\text {jup }}\right)$. One year later, the same team (Chauvin et al. 2005) measured the proper motion and verified that the brown dwarf and the companion were moving together, confirming the nature of this object, the first planet whose detection had been achieved by direct imaging.

The 2M1207 system is full of surprises, since the brown dwarf is still undergoing active accretion as shown by Mohanty et al. (2003) from high-resolution optical spectra around the $\mathrm{H} \alpha$ lines. It harbors a circumsubstellar disk, seen as a mid-IR excess by Sterzik et al. (2004), which can be explained by optically thick dust disk models. Rioz et al. (2006) have also derived the IR excess by using Spitzer data. However, no radio emission has been detected (Osten \& Jayawardhana 2006). Mohanty (2006, priv. comm.) has suggested that the secondary - the planetary mass companion - might have a disk too. Age is a key parameter in understanding the nature of the faint companion and the properties of the system.

In the present analysis, we will present in Sect. 2 the new spectroscopic data collected for several among the lowest mass candidate members of the association (two M8 and a L5), together with an estimate of the age and masses. Section 3 will revisit the data regarding the known members of the association, to estimate an accurate age by using different methods. We will end the paper with the conclusions and a discussion about the limits of the age-estimate techniques. 


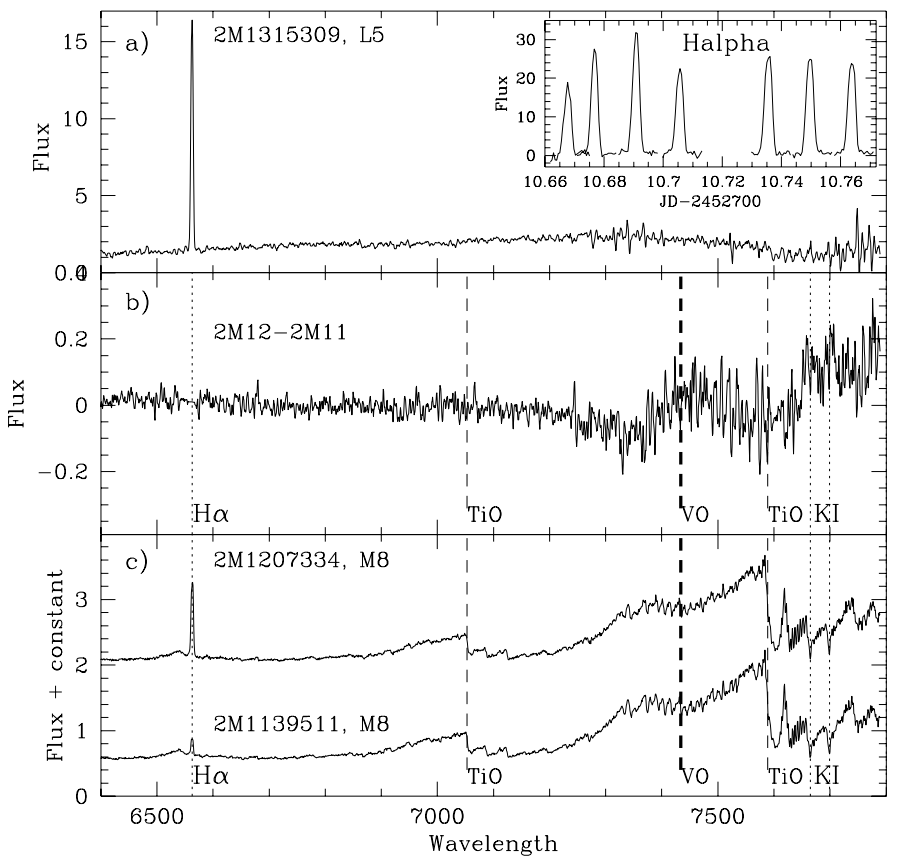

Fig. 1. Panel a) Medium-resolution spectrum of 2M1315. Panel c) Medium-resolution spectra of 2M1139 and 2M1207. Panel b) displays the result of the subtraction 2M1207-2M1139, after normalization at $7000 \AA$. Both objects are very similar, but 2M1207 has a flux excess in the red side of the spectrum, suggesting it might be somewhat cooler. Some excess in the blue side is apparent, which might be an effect of the accretion present in 2M1207. The inset in panel a displays the $\mathrm{H} \alpha$ profiles of 2M1315 (L5 spectral type). The continuum have been normalized using the flux at $7000 \AA$.

\section{Two M8 and a L5: 2M1139, 2M1207 and 2M1315}

\subsection{New spectroscopic observations: spectral classification}

We collected medium-resolution spectra of three low mass candidate members of TWA, namely 2M1139511-315921 (hereafter 2M1139), 2M1207433-393254 (2M1207), and 2M1315309-264951 (2M1315), with the Magellan/Baade telescope and the Boller \& Chivens (B\&C) spectrograph on March 11, 2003. We used the 1200 1/m gratings. The achieved spectral resolution is $R=2600$, as measured in the comparison arcs, with a spectral coverage of 6200-7800 A. During the observing run, among other targets, we observed 2M1139, 2M1207 and $2 \mathrm{M} 1315$, candidate members of the TW Hydrae association discovered by Gizis (2002) - see also Hall (2002a) in the case of 2M1315. The first two have been classified as M8 spectral type, whereas $2 \mathrm{M} 1315$ is much cooler, with a spectrum corresponding to L5. We collected six consecutive spectra of 2M1139, $600 \mathrm{sec}-$ onds each; four of $2 \mathrm{M} 1207$, with the same exposure time, and seven of $2 \mathrm{M} 1315$, the first one of $300 \mathrm{~s}$ and the others of $1200 \mathrm{~s}$ each. The data were reduced using standard techniques within IRAF $^{1}$. The individual spectra were combined using median algorithm in order to remove the cosmic rays. The final spectra can be seen in Fig. 1.

In addition to these objects, we observed a large number of spectral templates with spectral types in the interval K7-M9, both giants and dwarfs. More details regarding the

${ }^{1}$ IRAF is distributed by National Optical Astronomy Observatories, which is operated by the Association of Universities for Research in Astronomy, Inc., under contract to the National Science Foundation, USA. observations and the reduction process can be obtained in Barrado y Navascués et al. (2004c), Barrado y Navascués \& Jayawardhana (2004) and Barrado y Navascués et al. (2004b). For high-resolution spectroscopy of the two M8 targets, taken with Magellan/Baade and the echelle spectrograph MIKE, see Mohanty et al. (2003).

Based on the comparison with the spectral templates (namely HIP70669 -M6e-, vB07 -M7 V-, HIP68137 -M7-, HIP63642 M8III-, LHS2397 -M8V-, and BRI-0337-35 -M9), we have derived spectral types for 2M1139 and 2M1207. As can bee seen in Fig. 2 (only in the electronic version), where we display all these spectra (the solid lines correspond to the templates, whereas the dotted lines represent the TWA candidate members), the most similar templates are the M8V and M8III, the first case being closer to matching the target spectra. For comparison purposes, the spectra have been normalized in the area around $7500 \AA$. Therefore, it would be possible to classify these two objects as M8IV (they are contracting towards the Main Sequence). This classification is in agreement with Gizis (2002), who concluded that they have low gravity based on the VO indices measured from low-resolution spectroscopy, and by Mohanty et al. (2003) - from high-resolution spectroscopy. There are obvious differences between the M8 and M8 III templates and our TWA targets. See Sect. 2.2.2 for a further analysis concerning the luminosity class and the surface gravity.

We have also compared the spectra of 2M1139 and 2M1207 with each other, in other to verify whether they belong to the same spectral type. The visual inspection indicates that they are very similar, even when over-plotting both spectra. However, when the difference (one minus the other) or the ratio (one divided by the other) of both spectra is computed, 2M1207 might be slightly cooler that $2 \mathrm{M} 1139$. Figure $1 \mathrm{~b}$ displays the difference between both spectra (2M1207-2M1139), after normalizing them using the flux at $7000 \AA$. As a reference, we have plotted in the bottom panel the spectrum of 2M1139 and both panels indicate the location of key spectral features, such as the headband of $\mathrm{TiO}$ or the central wavelength of the VO. Two characteristics are evident from this graph: First, the flux of 2M1207 is greater than 2M1139 both in the red and blue part of the spectral range. This might be due to veiling - as clearly shown by Mohanty et al. (2003), 2M1207 is weakly accreting - and to a cooler spectral type. Second, the VO band is substantially different in both cases, being shallower at the central part and deeper at the edges in the case of 2M1207. Conversely, this can be interpreted as 2M1207 having a wider, shallower VO band. This might be related to differences in gravity (objects not exactly coeval) or temperature.

\section{2. $\mathrm{H} \alpha$ emission}

Since we have collected several consecutive spectra for each of these three candidate members of the TW Hydrae Association, it is possible in principle to search for variability in the $\mathrm{H} \alpha$ profile or equivalent width. This variability, if present, might be due to activity (both via modulation by the rotation or because of flares) or to accretion. We have not found any unambiguous evidence of variability in the case of 2M1139 or 2M1207. However, the range covered in time is very short, being only a fraction of the expected rotation period for this type of object. The measured equivalent widths are $W(\mathrm{H} \alpha)=10.2 \pm 0.7 \AA$ and $44.7 \pm 2.0 \AA$, respectively. Previous measurements were 10.0 and $300 \AA$, respectively, in low-resolution spectra (Gizis 2002), and 7.3 and $27.7 \AA$, for $2 \mathrm{M} 1139$ and 2M1207, in high-resolution spectra 
(Mohanty et al. 2003). More recently, Gizis \& Bharat (2004) measured an equivalent width of $42 \AA$ in the case of $2 \mathrm{M} 1207$. They speculate that Gizis (2002) collected the 2M1207 spectrum during a flare. However, although the flare might have taken place, these differences are to be expected, since, as noted by White \& Basri (2003), the higher the resolution, the smaller the equivalent width even if there are not intrinsic differences (more or less a factor of three going from low- to high-resolution). Scholz et al. (2005), by collecting a large number of high resolution spectra of 2M1207 in two different epochs separated by several weeks, have shown that the profile of the $\mathrm{H} \alpha$ line can change dramatically. The actual values can be found in Scholz \& Jayawardhana (2006).

2M1315, the L5 spectral type object, with a high proper motion and a possible age of more than $3 \mathrm{Gyr}$, is likely not a member of the association, as discussed by Hall (2002). Since it was observed during the same campaign as the two M8 TWA members, we have included the data.

In the case of $2 \mathrm{M} 1315$, some $\mathrm{H} \alpha$ variability might be present, although with some caveats. The continuum in this region, corresponding to its spectral type - L5 -, of this brown dwarf is very low, and the measurement of the equivalent width has a large uncertainty. The inset in Fig. 1a displays the $\mathrm{H} \alpha$ profiles for the seven independent spectra we have collected. The spectra have been normalized using the flux in the range around $7000 \AA$ (where the flux is maximum in the covered spectral range). The exposure times are 20 min except in the first spectrum, which is only $5 \mathrm{~min}$ long. The total time between the exposures is about $2.5 \mathrm{~h}$. As shown by Mohanty \& Basri (2003) and Zapatero Osorio et al. (2006), typical projected rotational velocities for L5 are in the range $20-40 \mathrm{~km} \mathrm{~s}^{-1}$. A L5 object has an effective temperature of about $1750 \mathrm{~K}$ (Basri et al. 2000), and for this temperature COND models from the Lyon group (Chabrier et al. 2000) are more appropriate. Depending on the assumed age (10 Myr or $5 \mathrm{Gyr}$ ), the expected rotation period is in the range 2.5-10.5 h (being shorter for older, faster objects). Therefore, we have covered just one rotation period in the optimal case, and we do not see any obvious modulation, although the data are too sparse.

The average equivalent width is $W(\mathrm{H} \alpha)=153 \pm 26 \AA$. Gizis (2002) measured $W(\mathrm{H} \alpha)=97 \AA$ in a low-resolution spectrum, and Hall $(2002 a, b)$ has reported values of 25,121 and $124 \AA$. The data suggest that we are dealing with a low mass object with frequent flaring, although other scenarios, such as acretion in a much younger object, cannot be ruled out.

\subsection{The age of $2 M 1139$ and $2 M 1207$}

\subsubsection{The lithium depletion and the age}

Both 2M1139 and 2M1207 show the lithium doublet at $6708 \AA$, as can be seen in Fig. 3. In addition, HeI6678 $\AA$, another accretion indicator, can be clearly seen in the second object, with an equivalent width of $W(\mathrm{HeI})=0.92 \pm 0.15 \AA$.

The measured lithium equivalent widths are $W(\mathrm{LiI})=0.62 \pm$ 0.13 and $0.51 \pm 0.09 \AA$, for $2 \mathrm{M} 1139$ and $2 \mathrm{M} 1207$, respectively. The comparison of these values with those of similar objects belonging to other young clusters or star forming regions (SFRs) indicate that this element has not been depleted so far in the two TW Hydra candidate members. In principle, depending on the effective temperature scale and the theoretical models, this fact imposes a maximum age of about $~ 50 \mathrm{Myr}$. The lithium abundance can be derived using the curve of growth (for example Zapatero Osorio et al. 2002), the measured

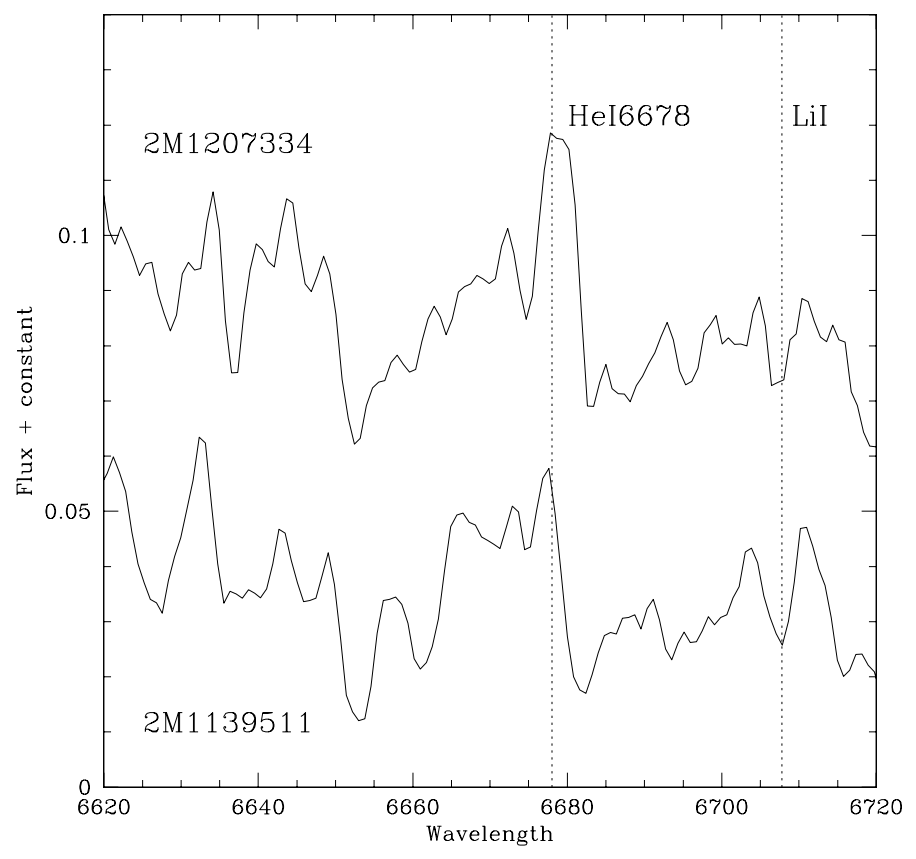

Fig. 3. Spectra around the HeI6678 and LiI6708 A features.

equivalent width and an estimate of the effective temperature (i.e., Luhman et al. 1999, $2720 \mathrm{~K}$ for M8 with luminosity class in between III and IV). The derived abundances are $A(\mathrm{Li})=3.10$ and 2.18 for $2 \mathrm{M} 1139$ and $2 \mathrm{M} 1207$, respectively -on a scale where $A(\mathrm{Li})=12+\log [N(\mathrm{Li}) / N(\mathrm{H})]$. The low abundance of 2M1207 can be interpreted as the presence of moderate veiling, due to accretion. Further details on the lithium depletion, the spread of the estimated abundance and age are given in Sect. 3.5.

Our spectrum of the L5 2M1315 is not good enough to even detect unambiguously the lithium feature, although it must be present due to its spectral type and estimated mass range, even if it does not belong to the association and it is much older. According to models by Chabrier et al. (2000), it should have a mass in the range $0.01-0.07 M_{\odot}$, for ages ranging from $10 \mathrm{Myr}$ to 5 Gyr. In any case, lithium cannot be used to establish the membership status of 2M1315 or to derive an age estimate for it.

\subsubsection{The surface gravity, the mass and the age}

The distances to 2M1139 and 2M1207, to the best of our knowledge, have not been measured so far by obtaining trigonometric parallaxes. Therefore, it is not possible to locate these objects in color-color or HR diagrams, without any assumption regarding their distance. However, Mamajek (2005) has derived a distance based on the moving cluster method.

It is possible to use the effective temperature and the surface gravity to estimate both the age and the mass of these two objects. This method is independent of the distance and does not require one to prove that they belong to the TW Hydrae Association. Figure 4 (only in the electronic version) displays a comparison between the spectra of 2M1139 and 2M1207 with several low mass stars in the spectral range around $7500 \AA$. These range includes a VO and a TiO bands and the KI7700 doublet. This alkali feature is gravity sensitive and can be used to estimate the surface gravity. Based on the diagram, the closest templates are HIP63642 (M8III), LHS2397a (M8V) and BRI0337-35 (M9). However, small differences persist for both the continuum and the KI7700 doublet. We have estimated that the 


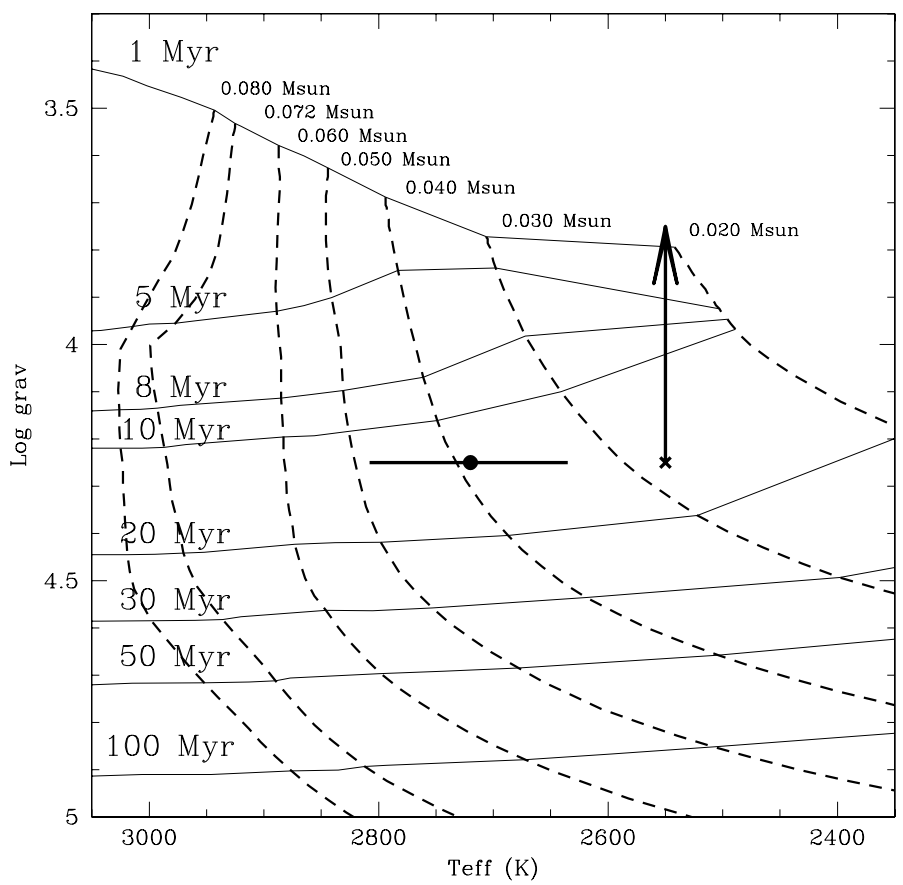

Fig. 5. The age for $2 \mathrm{M} 1207$, as derived from the surface gravity and the effective temperature (i.e., independent of the distance). The solid circle represents $T_{\text {eff }}$ derived from Luhman (1999) scale and gravity estimated by comparison with spectral templates. The arrow/cross indicates the location when the temperature derived by Mohanty (2006, priv. comm.) is used. The isochrones and tracks correspond to models by Baraffe et al. (1998).

luminosity class has to be in between III and V, with an intermediate gravity, somewhat closer to V than to III $(\log g=4.25 \pm 0.5)$.

The comparison of the derived surface gravity and effective temperature with theoretical models is presented in Fig. 5 (solid and dashed lines for isochrones and tracks, respectively). The models correspond to Baraffe et al. (1998) and the effective temperature to the average between giants and dwarfs by Luhman (1999). Note that other scales, such as dwarfs by Bessell (1991), Leggett (1992), or Luhman (1999), would give temperatures for 2M1139 and 2M1207 about 100-150 K cooler, providing smaller masses and ages slightly older. However, the temperature scale by Luhman (1999) has been derived to match the Baraffe et al. (1998) models. Figure 5 indicates that the masses of these two objects are $0.04 \pm 0.01 M_{\odot}$, and the age slightly younger than $15 \mathrm{Myr}$, although the uncertainty in the derived surface gravity allows for much younger or older ages (from 1 to $30 \mathrm{Myr}$ ); a more realistic lower limit would be $5 \mathrm{Myr}$.

Spectral synthesis in young brown dwarfs produces effective temperatures about $200 \mathrm{~K}$ cooler than the values derived from the scale of Luhman (1999). An example corresponding to the young brown dwarf LS-RCrA 1 can be found in Barrado y Navascués et al. (2004c). In the case of 2M1207, a effective temperature of about $2550 \mathrm{~K}$ can be derived (Mohanty, priv. comm.). The same analysis produces a value of the gravity of $\log g=4.0 \pm 0.5$. These quantities would locate $2 \mathrm{M} 1207$ on top of younger isochrones, with a maximum age of $25 \mathrm{Myr}$ and a probable age of $8 \mathrm{Myr}$.

The figure shows that these two objects are bona fide young brown dwarfs, independently of their belonging to the TW Hydrae Association.

\section{Revisiting the TW Hya association}

\subsection{The data}

One of the goals of this paper is to derive an accurate age for the TW Hydrae Association. To do so, we have collected data from the literature. So far, there are a few dozen candidate members of the association. However, we have restricted ourselves to those whose parallaxes have been measured, or whose properties have been studied in depth (lithium and $\mathrm{H} \alpha$ equivalent widths, and so on). There are several age indicators that have been used, such as the rotational velocity, the orbital period or the coronal activity as measured from X-rays. However, they have a very large scatter for a particular spectral type and they cannot provide an age estimate accurate enough for our purposes. For this reason, we have only studied the location in a HR diagram, the lithium and the $\mathrm{H} \alpha$ emission.

Among the papers we have used to extract data are: Rucinski \& Krautter (1983), de la Reza et al. (1989), Jura et al. (1993), Stauffer et al. (1995), Soderblom et al. (1996), Hoff et al. (1998), Sterzik et al. (1999), Webb et al. (1999), Neuhauser et al. (2000), Torres et al. (2000), Muzerolle, priv. comm (2000), Zuckerman et al. (2001), Gizis (2002), Song et al. (2002), Mohanty et al. (2003), Reid et al. (2003), Song et al. (2003), Torres et al. (2003), Gizis et al. (2004), Weinberger et al. (2004), Zuckerman \& Song (2004), Scholz \& Jayawardhana (2006), Jayawardhana et al. (2006), and Scholz et al. (2006). In this paper we will use Hipparcos distances (ESA 1987). However, Mamajek (2005), using the moving cluster method, has been able to derive an individual distance for $2 \mathrm{M} 1207$ (56 $\pm 6 \mathrm{pc}$ ) and for the association as a whole (49 pc). The data are listed in Table 1.

The bolometric luminosities were derived from the magnitudes in different bands (namely $V, I, J$ and $K \mathrm{~s}$ ), the Hipparcos parallax and the bolometric corrections for each band. The latter values were computed using the expressions provided by Schmidt-Kaler and the spectral type for $V$; Bessell (1991) and the color index $(I-K)$ for $I$; Lawson (1996) and the spectral type for $J$; and Tinney (1993) and the color index $(I-K)$ for $K$ s.

$M_{\mathrm{bol}}=5+5 \log \pi+\mathrm{M}($ band $)+\mathrm{BC}($ band $)$

$L_{\mathrm{bol}}=10^{-0.4\left(M_{\mathrm{bol}}-M_{\mathrm{bol}}^{\odot}\right)}\left(L / L_{\odot}\right)$

where $M_{\mathrm{bol}}{ }^{\odot}$ is the bolometric magnitude of the Sun and has been assumed to be $4.75 \mathrm{mag}$. Errors in the bolometric luminosity were estimated adding in quadrature those produced by the errors in the distance and those coming from either the photometry - for the individual values coming from each band - or the dispersion from the average of those values.

\subsection{The color-magnitude diagram: membership and an initial age estimate}

Figure 6 represents an infrared color-magnitude diagram (CMD), and includes evolutionary tracks and isochrones by Baraffe et al. (1998), as well as the location of several TW Hydrae candidate members whose parallaxes have been measured. The $J$ and $K$ s magnitudes come from the 2MASS All Sky Survey (Cutri et al. 2003). Although IR CMDs are not the best ones to derive masses and ages, the optical photometry of these objects was not derived in a homogeneous way or simultaneously for all members. Therefore, we have preferred to use the 2MASS data as a first approach to asses the membership of this handful of candidates. Errorbars include the error coming from the photometry and the distance, added in quadrature. This diagram clearly indicates that HIP53486 and HIP50796, which have been proposed by Makarov \& Fabricius (2001), 


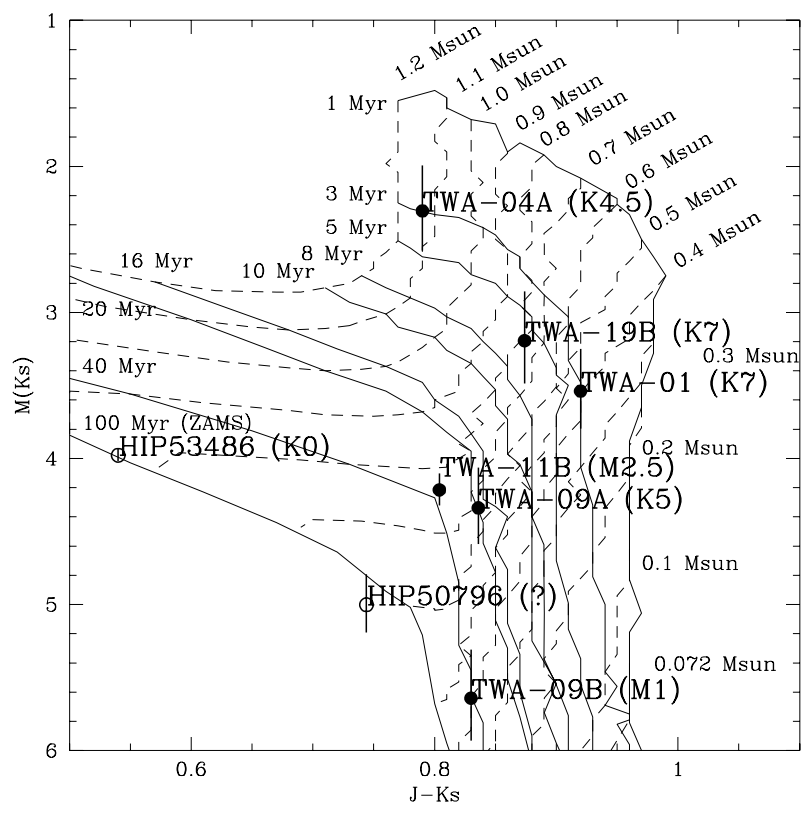

Fig. 6. Infrared color-color diagram, showing models by Baraffe et al. (1998) and IR photometry from 2MASS.

Song et al. (2002) and Torres et al. (2003), are not members of the association, being too old. Moreover, the visual binary TWA09 might not belong to the association either (see next subsection). Based on this figure, an age about 3-8 Myr can be estimated. As noted by the authors, colors for the Baraffe et al. (1998) and other theoretical isochrones are not certain. They recommend the use of bolometric luminosities instead.

\subsection{The Hertzprung-Russell diagram: an accurate age}

The location of those TW Hydrae members in a HR diagram is presented in Figs. 7-9 (only in the electronic version). In the case of the four panels in Fig. 7, we have displayed models by Baraffe et al. (1998); the effective temperature was derived using the scale by Luhman (1999) (average between III and V luminosity classes), but in each panel the bolometric luminosity was derived using a different method (see Sect. 3.1). Thus, they provide an estimate of the error introduced by the conversion from magnitudes into luminosities. In the case of the three panels of Fig. 8, we have only changed the temperature scale, and we have included values derived from scales by Bessell (1991), Leggett (1992) and Luhman (1999). Figure 9 provides the effect on the age of different theoretical models. Regardless of the specific details (the model, the temperature or the method used to derive luminosities), we believe that the age is well constrained by the 3 and $20 \mathrm{Myr}$ isochrones, with an optimal value of $8 \mathrm{Myr}$.

The location of TWA09 seems to indicate that this object is a spurious member of the association. This result agrees with recent estimates by de la Reza et al. (2006), who studied the dynamical evolution of the association, showing that it has a different orbit around the Galaxy compared to other members.

\subsection{The $\mathrm{H} \alpha$ emission, the fraction of accreting members and the age}

In Barrado y Navascués \& Martín (2003) - see also Barrado y Navascués et al. (2004a) - we have shown that the $\mathrm{H} \alpha$ equivalent width, as measured using low-resolution spectroscopy, is a powerful criterion from the statistical point of view to classify

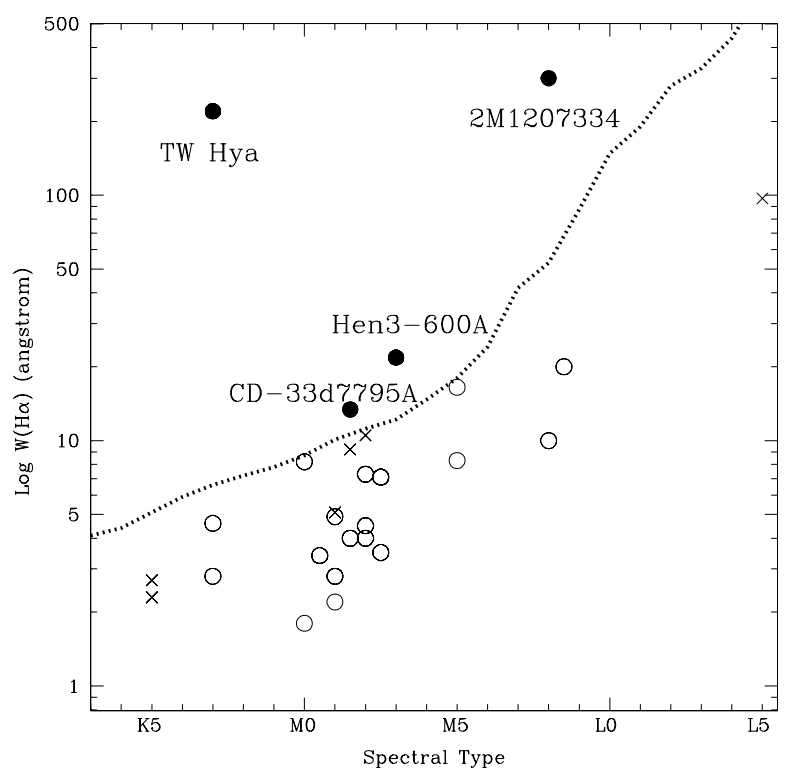

Fig. 10. $\mathrm{H} \alpha$ equivalent width - in logarithm - versus the spectral type. The dotted line is the accretion criterion proposed by Barrado y Navascués \& Martín (2003). Classical and weak-line/post T Tauri stars or substellar analogs are displayed with solid or open circles, respectively. Probable non-members are included as crosses.

accreting and non-accreting objects, since the saturation of the chromospheric activity at $\log \{\operatorname{Lum}(\mathrm{H} \alpha) / \operatorname{Lum}($ bol $)\}=-3.3$, which appears in members of young open clusters, provides a clear discrimination between Classical T Tauri stars (and substellar analogs) and Weak-line T Tauri stars (or post-T Tauri stars). We have used this criterion with the TW Hydrae Association. Figure 10 displays the $\mathrm{H} \alpha$ equivalent width versus the spectral type. Solid circles represent low mass stars and brown dwarfs which are undergoing accretion and/or having circunstellar disks (as measured using high-resolution spectroscopy and/or infrared excess), whereas open circles correspond to nonaccreting objects. Other TWA candidate members, whose membership is dubious, appear as crosses. As can be seen, the criterion is fulfilled in all cases: those objects having $W(\mathrm{H} \alpha)$ larger than the saturation limit do have accretion and those below it do not show any evidence of it.

We have derived the ratio between accreting and the total number of members. To avoid statistical problems with low numbers, we have restricted ourselves to the stellar domain. Depending on how the visual and spectroscopic binaries and multiple systems are accounted for and including errors (computed as Poisson noise), this ratio is between 0.20 and 0.0625 , with an optimum value of 0.13 . We have derived this ratio for several well known star forming regions and very young clusters (see details in Barrado y Navascués \& Martín 2003) and compared them with the result for the TW Hydrae Association. Figure 11 summarizes this comparison. The solid, thick line represents the behavior of the SFRs, whereas the best value and upper/lower limits for the TWA appear as a dashed line and two dotted lines, respectively. If we assume that TWA follows the trend seen in the SFRs, we can estimate the age of the former group as $7_{-3}^{+8}$ Myr.

\subsection{Lithium depletion and age}

Our final criteria to estimate the age of the TWA are the lithium equivalent width and abundance. First, we have compared the 


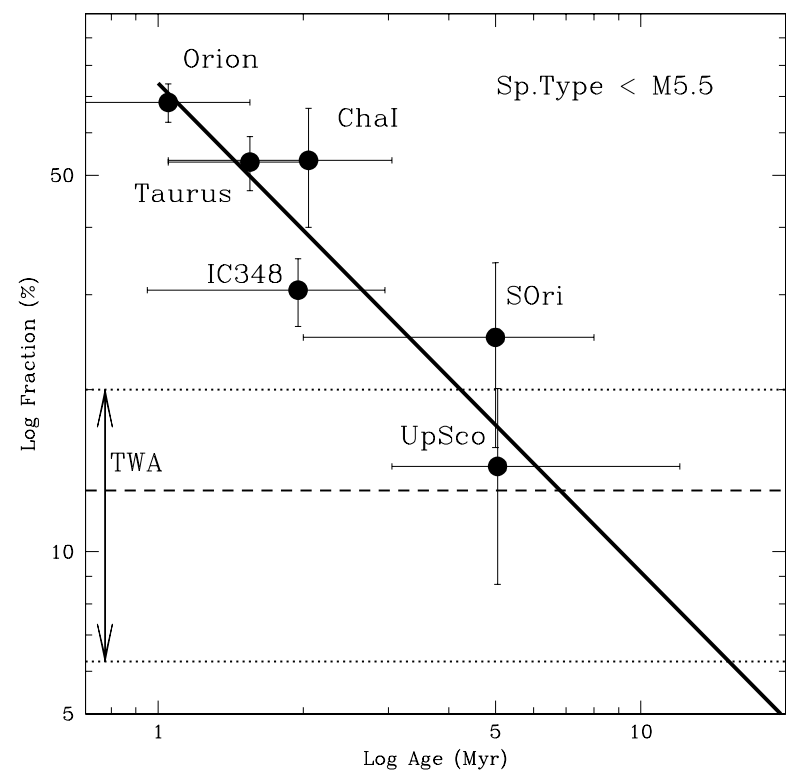

Fig. 11. Fraction of stars classified as CTT stars (based on the $\mathrm{H} \alpha$ equivalent width) for different star forming regions and young clusters. The dashed line corresponds to the average value for TWA, whereas the two dotted lines represent the maximum and minimum value. The clear trend with the age (solid line) indicates that TWA is about 7 Myr old.

$W($ LiI) with several young clusters and SFRs. For simplicity, we only show in Fig. 12 a comparison with the Lambda Orionis open cluster (Collinder 69). The data come from Dolan \& Mathieu (1999, 2001) and Barrado y Navascués et al. (2006, in preparation, see this paper for details). In this diagram, the solid line corresponds to the upper envelope of the values measured in young open clusters (with ages in the range 30-150 Myr). The long-dashed line delimits the areas for weak-line and post-T Tauri stars (adapted from Martín 1997 and Martín $\&$ Magazzù 1999). We have computed the lithium depletion isochrones using the temperatures and abundances listed in the models by Baraffe et al. (1998), the curves of growth of Zapatero Osorio et al. (2002) and the effective temperature for luminosity class IV by Luhman (1999). These lithium isochrones are represented as thick, short-dashed lines in the figure $(1,8,10$, 15 and $20 \mathrm{Myr}$ ). Note that on the $1 \mathrm{Myr}$ isochrone, lithium has not been depleted, and still has an abundance of $A(\mathrm{Li})=3.1$, the cosmic value. In Fig. 12, TWA members are displayed as solid circles (CTT), open circles (WTT/PTT) and crosses (probable non-members). The asterisks correspond to members of the Lambda Orionis cluster located in the central area (after Dolan \& Mathieu 1999, 2001; and Barrado y Navascués et al. 2004b). As shown by Dolan \& Mathieu (1999, 2001, 2002), the Lambda Orionis cluster is about $5 \mathrm{Myr}$. It cannot be older than $8 \mathrm{Myr}$, since the star $\lambda^{1}$ itself, a O8 III, with a mass estimate of about $30 M_{\odot}$ (Barrado y Navascués et al. 2005) would have exploded as a supernova in that case.

The $W(\mathrm{Li})$ presents a clear scatter for mid-M stars and brown dwarfs (this also happens for other spectral ranges). The origin of this phenomenon is not clear but it can be related to rotation, activity, or a low $\mathrm{S} / \mathrm{N}$ - i.e., large errors. Most of the objects in the Lambda Orionis cluster are clustered around the loci of $A(\mathrm{Li})=3.1$ (i.e., the cosmic abundance, undepleted). A substantial fraction of TWA members have $W(\mathrm{Li}) \mathrm{s}$ smaller than the values corresponding to the cosmic abundance and might have undergone some depletion. On the other hand, some veiling might be present in 2M1207 (see Sect. 2.2.1). Therefore, this figure

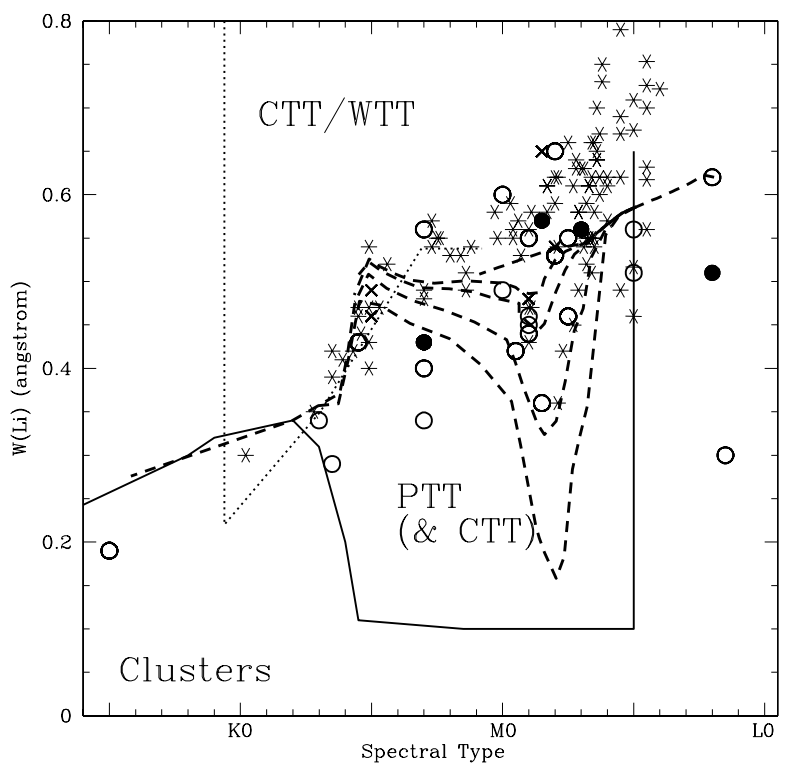

Fig. 12. Lithium equivalent with versus the spectral type. The solid line corresponds to the upper envelope of the values measured in young open clusters such as IC2391, IC2602, the Pleiades and M35. The long-dashed line delimits the areas for weak-line and post-T Tauri stars (adapted from Martín 1997 and Martín \& Magazzù 1999). Thick, short-dashed lines correspond to Baraffe et al. (1998) lithium depletion isochrones (using curves of growth from Zapatero Osorio et al. 2002 and the temperature scale by Luhman 1999). We have represented values corresponding to $1,8,10,15$ and $20 \mathrm{Myr}$ isochrones. The first one has no lithium depletion $-A(\mathrm{Li})=3.1$. TWA members are displayed as solid circles (CTT), open circles (WTT/PTT) and probable non-members (crosses). The asterisks correspond to members of the Lambda Orionis cluster (after Dolan \& Mathieu 1999, 2001; and Barrado y Navascués et al. 2006). This cluster is about 5 Myr, with a maximum age of $8 \mathrm{Myr}$. Based on the lithium equivalent width, the TW Hydrae association is older than the Lambda Orionis cluster.

indicates that TWA is older than the Lambda Orionis cluster. The same result can be achieved with other clusters, such as the Sigma Orionis cluster (3-5 Myr). The comparison with the theoretical lithium depletion isochrones indicate that the TWA is younger that $15 \mathrm{Myr}$, with an average age of $10 \mathrm{Myr}$.

We have also derived lithium abundances for the TWA members, and compared these values with the prediction from different models (D'Antona \& Mazzitelli 1997; Baraffe et al. 1998; Siess et al. 2000). To do so we have used the curves of growth of Zapatero Osorio et al. (2002) and different temperature scales (Bessell 1991; Leggett 1992; Luhman 1999). These comparisons are presented in nine panels (Fig. 13, only in the electronic version). Accreting objects, that might have a $W(\mathrm{Li})$ and therefore a $A(\mathrm{Li})$ affected by accretion (because of the veiling) are represented as solid circles, whereas non-accreting objects appear as open circles. Dubious members are included as crosses. Note the large scatter for any particular comparison; even the non-accreting objects are far from being in a lithium depletion isochrone. With some caveats, the age can be estimated as $8 \mathrm{Myr}$, with lower and upper limits of 3 and $15 \mathrm{Myr}$.

\section{Discussion and conclusions}

The TW Hydrae association is becoming a cornerstone in our understanding of phenomena such as stellar accretion and the formation of planetary systems, due to the fact that it contains several dozen candidate members (only a fraction of them 
Table 2. Summary of the age estimates (in Myr).

\begin{tabular}{lrrr}
\hline \hline Method & Minimum & Age & Maximum \\
\hline Log grav $^{1}$ & 3 & 15 & $30^{2}$ \\
CMD, parallax $^{3}$ & 3 & 5 & 8 \\
HRD, parallax $^{3}$ & 3 & 8 & 20 \\
Disk fraction & 4 & 7 & 15 \\
W(H $\alpha)$ & 4 & 7 & 15 \\
W(Li) & 5 & 10 & 15 \\
A(Li) & $\sim 3$ & $\sim 8$ & 15 \\
\hline
\end{tabular}

${ }^{1}$ Only for $2 \mathrm{M} 1139$ and $2 \mathrm{M} 1207 .{ }^{2}$ Based on spectral synthesis, the upper limit for the age is $15 \mathrm{Myr}$, with $T_{\text {eff }}=2550 \mathrm{~K} .{ }^{3}$ Only for those having parallax.

confirmed), is nearby, and its age is critical for the evolution of several stellar properties. Our main goal has been to establish an accurate age for the whole association and, in the process, for the brown dwarf 2M1207, an accreting member which contains, at least, a planetary mass object located at a projected distance of about $~ 50 \mathrm{AU}$.

We have used the available data for the bona-fide members and compared them with the properties of well-known star forming regions and young clusters, as well as theoretical models. The methods we have used include HR and color-magnitude diagrams, surface gravity, activity, and lithium. Table 2 summarizes all the age estimates, including minimum and maximum values. Depending on the method (the property we have used to estimate the age), the derived values are in the range of $5 \mathrm{Myr}$ (color-magnitude diagram with 2MASS data and theoretical models, not very reliable) to $15 \mathrm{Myr}$ (for the gravity). The "best" value is 10 Myr. The minimum age is imposed by color-magnitude and HR diagrams, as well as by the lithium abundance, whereas an upper limit of $20 \mathrm{Myr}$ can be derived from the HR Diagram. Therefore, we can say that the age of the association is $10_{-7}^{+10} \mathrm{Myr}$.

In the case of $2 \mathrm{M} 1207$, the gravity, as measured by midresolution optical spectroscopy, and its comparison with theoretical models by Baraffe et al. (1998) indicates that the age is $15_{-10}^{+15} \mathrm{My}$, in agreement with the age of the association. As a comparison, Mamajek (2005) derived an age for TWA based on the moving cluster distance method, an expansion age. After confirming the membership of 2M1207, he obtained a lower limit of $10 \mathrm{Myr}$. This value is in contradiction with an estimation based on the dynamical evolution of the association (Makarov \& Fabricius 2001; de la Reza et al. 2006), 8.3 0.8 Myr. Moreover, Makarov et al. (2005) have found that three members (TWA 1, 4 and 11) might be younger, since they derived an expansion age of $4.7 \pm 0.6 \mathrm{Myr}$ for them. However, our own estimation is compatible with these determinations.

In this analysis, we have assumed the coevality of the TW Hydrae Association. This is an hypothesis which might not be true. Lawson \& Crause (2005) have found a bimodal distribution in the rotation periods, and their interpretation is that there are two distinct populations, which are 10 and 17 Myr old. As we have shown, the almost identical 2M1207 and 2M1139 show small differences in their spectrum, which might be due to different gravities, which would indicate different ages. Therefore, non-coevality is a possibility.

We have several caveats regarding these age estimations. First, age estimation is difficult. Each individual method has its own problems. Some among them depend on the direct comparison with theoretical models (such as the HR diagram), other include not well-understood properties (such as the process of accretion and its evolution evolution or the lithium abundance). There are also problems transferring observational into theoretical quantities, such as from magnitudes and colors into effective temperatures and luminosities (Stauffer et al. 1995). Moreover, colors can be affected by activity and related effects (see the case of the Pleiades, Stauffer et al. 2003). On the other hand, different methods can provide very different ages. A case study is lithium depletion chronology for young clusters such as NGC2547, IC2391, Alpha Per and the Pleiades, which produces ages about 50\% larger (Stauffer et al. 1998, 1999; Barrado y Navascués et al. 1998, 1999a, 2004d; Oliveira et al. 2003) than canonical values, as derived from isochrone fitting in the lower Main Sequence or the turn-over method.

Therefore, our age - or any age - estimation should be understood in the context of a particular age scale, in an evolutionary sequence. The important fact is that we can sort different star forming regions, moving groups, associations and open clusters by their age, stating, for instance, that Taurus is younger than Lambda Orionis, which is younger than IC2391. We can be sure that TWA is in between these two latter clusters. Since we have analyzed several associations, trying to understand different, although related, phenomenology, in the same way (see, for instance, the case of protoplanetary disks of $\beta$ Pic and HR4796 and Fomalhaut (Barrado y Navascués et al. 1999b, 2001, and references therein), we are trying to link a whole array of properties in a coherent, but relative, evolutionary context. However, much work remains to be done.

An absolute age-scale (or scales), based on homogeneous datasets (same methodology to acquire and analyze the data), is needed, coupled with theoretical work, in order to reach an agreement between the estimates provided by different properties.

Acknowledgements. Based on data collected by the Magellan telescopes. D.B.y.N. is indebted to the Spanish "Programa Ramón y Cajal" ESP2004-01049 and S0505/ESP/0361 and to N. Huelamo, R. Jayawardhana and S. Mohanty. The comments of the referee, M. Sterzik, have been very helpful. This publication makes use of data products from the Two Micron All Sky Survey.

\section{References}

Baraffe, I., Chabrier, G., Allard, F., \& Hauschildt, P. H. 1998, A\&A, 337, 403 Barrado y Navascués, D. 2001, Young Stars Near Earth: Progress and Prospects, ed. Ray Jayawardhana \& Thomas Greene (San Francisco: Astronomical Society of the Pacific), ASP Conf. Ser., 244, 63

Barrado y Navascués, D. 2004, A\&A, 422, 631

Barrado y Navascués, \& Martín, E. L. 2003, AJ, 126, 2997

Barrado y Navascués D., \& Jayawardhana R. 2004, ApJ, 615, 840

Barrado y Navascués, D., Stauffer, J. R., \& Bouvier, J. 1998, Ap\&SS, 263, 239

Barrado y Navascués, D., Stauffer, J. R., \& Patten, B. 1999a, ApJ, 522, L53

Barrado y Navascués, D., Stauffer, J. R., Song, I., \& Caillault, J.-P. 1999b, ApJ, $520, \mathrm{~L} 123$

Barrado y Navascués, Martín, E. L., Jayawardhana, R. J., \& Mohanty, S. 2004a, Kluwer Academic Publishers (Magnetic Fields)

Barrado y Navascués, D., Stauffer, J. R., et al. 2004b, ApJ, 610, 1064

Barrado y Navascués, D., Mohanty, S., \& Jayawardhana, R. 2004c, ApJ, 604, 284

Barrado y Navascués, D., Stauffer, J. R., \& Jayawardhana, R. 2004d, ApJ, 614, 386

Barrado y Navascués, D., Stauffer, J. R., \& Bouvier, J. 2005, The initial mass funtion 50 years later, ed. E. Corbelli, F. Palle, \& H. Zinnecker, Ap\&SS Library (Dordrecht: Springer), 327, 133

Barrado y Navascués, et al. 2006, ApJ, in prep.

Basri, G., Mohanty, S., Allard, F., et al. 2000, ApJ, 538, 363

Bessell, M. S. 1991, AJ, 101, 662

Chauvin, G., Lagrange, A.-M., Dumas, C., et al. 2004, A\&A, 425, L29

Chabrier, G., Baraffe I., Allard, F., \& Hauschildt, P. 2000, ApJ, 542, L119

Chauvin, G., Lagrange, A.-M., Dumas, C., et al. 2005, A\&A, 438, L25

Cutri, R. M., et al. 2003, 2MASS All-Sky Catalog of Point Sources, University of Massachusetts and Infrared Processing and Analysis Center, (IPAC/California Institute of Technology). 
D’Antona, F., \& Mazzitelli, I. 1997, in Cool Stars in Clusters and Associations, ed. R. Pallavicini, \& G. Micela, Mem. Soc. Astron. It., 68 (4), 807

de la Reza, R., Torres, C., Quast, G., Castilho, B., \& Vieira, G. 1989, ApJ, 343, L61

de la Reza, R., Jilinski, E., \& Ortega, V. G. 2006, AJ, 131, 2609

Dolan, C. J., \& Mathieu, R. D. 1999, AJ, 118, 2409

Dolan, C. J., \& Mathieu, R. D. 2001, AJ, 121, 2124

Dolan, C. J., \& Mathieu, R. D. 2002, AJ 123, 387

ESA, The Hipparcos Catalogue, 1987 ESA SP-1200

Favata, F., Barbera, M., Micela, G., \& Sciortino, S. 1995, A\&A, 295, 147

Gizis, J. E. 2002, ApJ, 575, 484

Gizis, J. E., \& Bharat, R. 2004, ApJ, 608, L113

Hall, P. B. 2002a, ApJ, 564, L89

Hall, P. B. 2002b, ApJ, 580, L77

Hoff, W., Henning, T., \& Pfau, W. 1998, A\&A 336, 242

Jayawardhana, R., Coffey, J., \& Scholz, A. 2006, ApJ, in press [arXiv: astro-ph/0605601]

Jensen, E. L. N., Cohen, D. H., \& Neuhauser, R. 1998, AJ, 116, 414

Jura, M., Zuckerman, B., Becklin, E. E., \& Smith, R. 1993, ApJ, 418, L37

Jura, M., Malkan, M., White, R., Telesco, C., Pina, R., \& Fisher, R. 1998, ApJ, 505,897

Kastner, J., Zuckerman, B., Weintraub, D., \& Forveille, T. 1997, Science, 277, 67

Lawson, W. A., \& Crause, L. A. 2005, MNRAS, 357, 1399

Lawson, W. A., Feigelson, E. D., \& Huenemoerden, D. P. 1996, MNRAS, 280, 1071

Leggett, S. K. 1992, ApJS, 82, 351

Lowrance, P. J., et al. 1999, ApJ, 512, L69

Luhman, K. 1999, ApJ, 525, 466

Makarov, V. V., \& Fabricius, C. 2001, A\&A, 368, 866

Makarov, V. V., Gaume, R. A., \& Andrievsky, S. M. 2005, MNRAS, 362, 1109

Mamajek, E. E. 2005, ApJ, 634, 1385

Martín, E. L. 1997, A\&A, 321, 492

Martín, E. L., \& Magazzù, A. 1999, A\&A, 342, 173

Masciadri, E., Mundt, R., Henning, Th., et al. 2004, ApJ, 625, 1004

Mohanty, S., \& Basri, G. 2003, ApJ, 583, 451

Mohanty, S., Jayawardhana, R., \& Barrado y Navascués, D. 2003, ApJ, 593 L109

Muzerolle, J., Calvet, N., Briceño, C., Hartmann, L., \& Hillenbrand, L. 2000, ApJ, 535, L47

Neuhauser, R., Guenther, E., Petr, M., et al. 2000, A\&A, 360, L39

Neuhauser, R., Guenther, E. W., Alves, J., et al. 2003, AN, 324, 543

Oliveira, J. M., Jeffries, R. D., Devey, C. R., et al. 2003, MNRAS, 342, 651

Osten, R. A., \& Jayawardhana, R. 2006, ApJL, in press

[arXiv:astro-ph/0604521]
Prato, L., Ghez Pina, R., Telesco, C., Fisher, R., et al. 2001, ApJ, 549, 590 Reid, N. 2003, MNRAS, 342, 837

Riaz, B, Gizis, J. E., \& Hmiel, A. 2006, ApJ, 639, L79

Rucinski, S. M., \& Krautter, J. 1983, A\&A, 121, 217

Scholz, A., \& Jayawardhana, R. 2006, ApJ, 638, 1056

Scholz, A., Jayawardhana, R., \& Brandeker, A. 2005, ApJ, 629, L41

Siess, L., Dufour, E., \& Forestini, M. 2000, A\&A, 358, 593

Soderblom, D., Henry, T., Shetrone, M., Jones, B., \& Saar, S. 1996, ApJ, 460 984

Soderblom, D., et al. 1998, ApJ, 498, 385

Song, I., Bessel, M., \& Zuckerman, B. 2002, A\&A, 385, 862

Song, I., Zuckerman, B., \& Bessell, M. 2003, ApJ, 599, 342

Stauffer, J. R., Hartmann, L., \& Barrado y Navascués, D. 1995, ApJ, 454, 910

Stauffer, J. R., Schultz, G., \& Kirkpatrick, J. D. 1998, ApJ, 499, L199

Stauffer, J. R., Barrado y Navascués, D., Bouvier, J., et al. 1999, ApJ, 527, 219

Stauffer, J. R., Jones, B. F., Backman, D., et al. 2003, AJ, 126, 833

Sterzik, M., Alcalá, J., Corvino, E., \& Petr, M. 1999, A\&A, 346, L41

Sterzik, M. F., Pascucci, I., Apai, D., van der Bliek, N., \& Dullemond, C. P. 2004, A\&A, 427, 245

Tachihara, K., Neuhauser, R., Frink, S., \& Guenther, E. 2003, AN, 324, 543

Tinney, C. G., Mould, J. R., \& Reid, I. N. 1993, AJ, 105, 1045

Torres, C., da Silva, L., Quast, G., de la Reza, R., \& Jilinski, E. 2000, AJ, 120, 1410

Torres Guenther, E., Marschall, L., Neuhauser, R., Latham, D., \& Stefanik, R. 2003, ApJ, 125, 825

Tsuboi, Y., Maeda, Y., Feigelson, E., Garmire, G., Chartas, G., et al. 2003, ApJ, 587, L51

Uchida, K. I., Calvet, N., Hartmann, L., Kamper, F., Forrest, W. J., et al. 2004, ApJS, 154, 349

Webb, R. A., Zuckerman, B., Platais, I., et al. 1999, ApJ, 512, L63

Weinberger, A., Becklin, E. E., Zuckerman B. M., \& Song, I. 2004, AJ, 127, 2246

Weintraub, D. A., Saumon, D., Kastner, J. H., \& Forveille, T. 2000, ApJ, 530, 867

White, R. J., \& Basri, G. 2003, ApJ, 582, 1109

Zapatero Osorio, M. R., Béjar, V. J. S., Pavlenko, Ya., et al. 2002, A\&A, 384, 937

Zapatero Osorio, M. R., Martín, E. L., Bouy, H., et al. 2006, ApJ, in press [arXiv: astro-ph/0603194]

Zuckerman, B., \& Becklin, E. E. 1993, ApJ, 406, L25

Zuckerman, B., Webb, R., Schwartz, M., \& Becklin, E. 2001, ApJ, 549, L233

Zuckerman, B., \& Song, I. 2004, ARA\&A, 42, 685 
D. Barrado y Navascués: The age of TWA and 2M1207, Online Material $p 1$

\section{Online Material}


D. Barrado y Navascués: The age of TWA and 2M1207, Online Material $p 2$
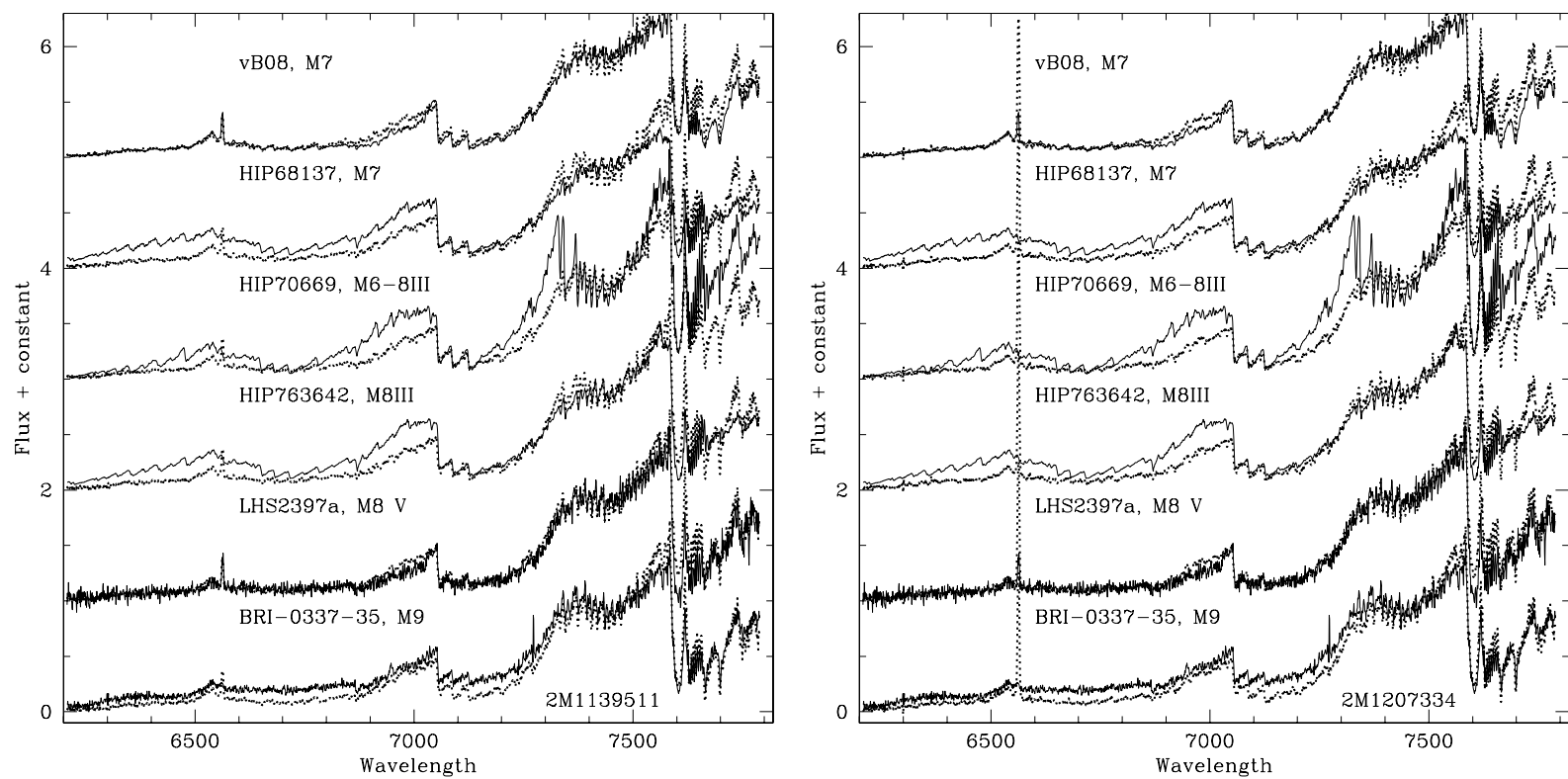

Fig. 2. Spectral classification of $2 \mathrm{M} 1139$ and $2 \mathrm{M} 1207$. We have normalized the spectra using the flux around $7450 \AA$.
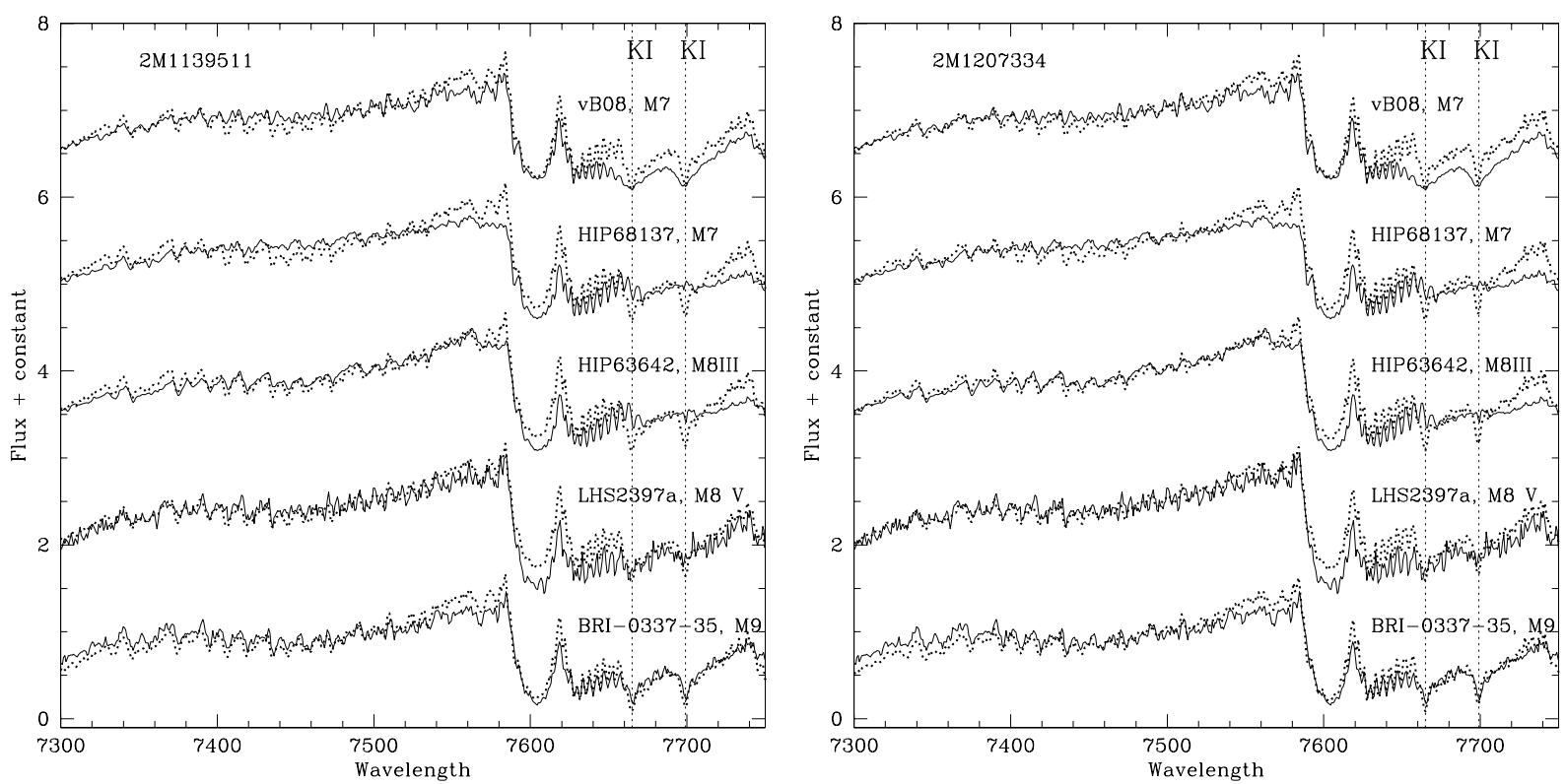

Fig. 4. Deriving the surface gravity by comparison with spectral templates. 
D. Barrado y Navascués: The age of TWA and 2M1207, Online Material $p 3$
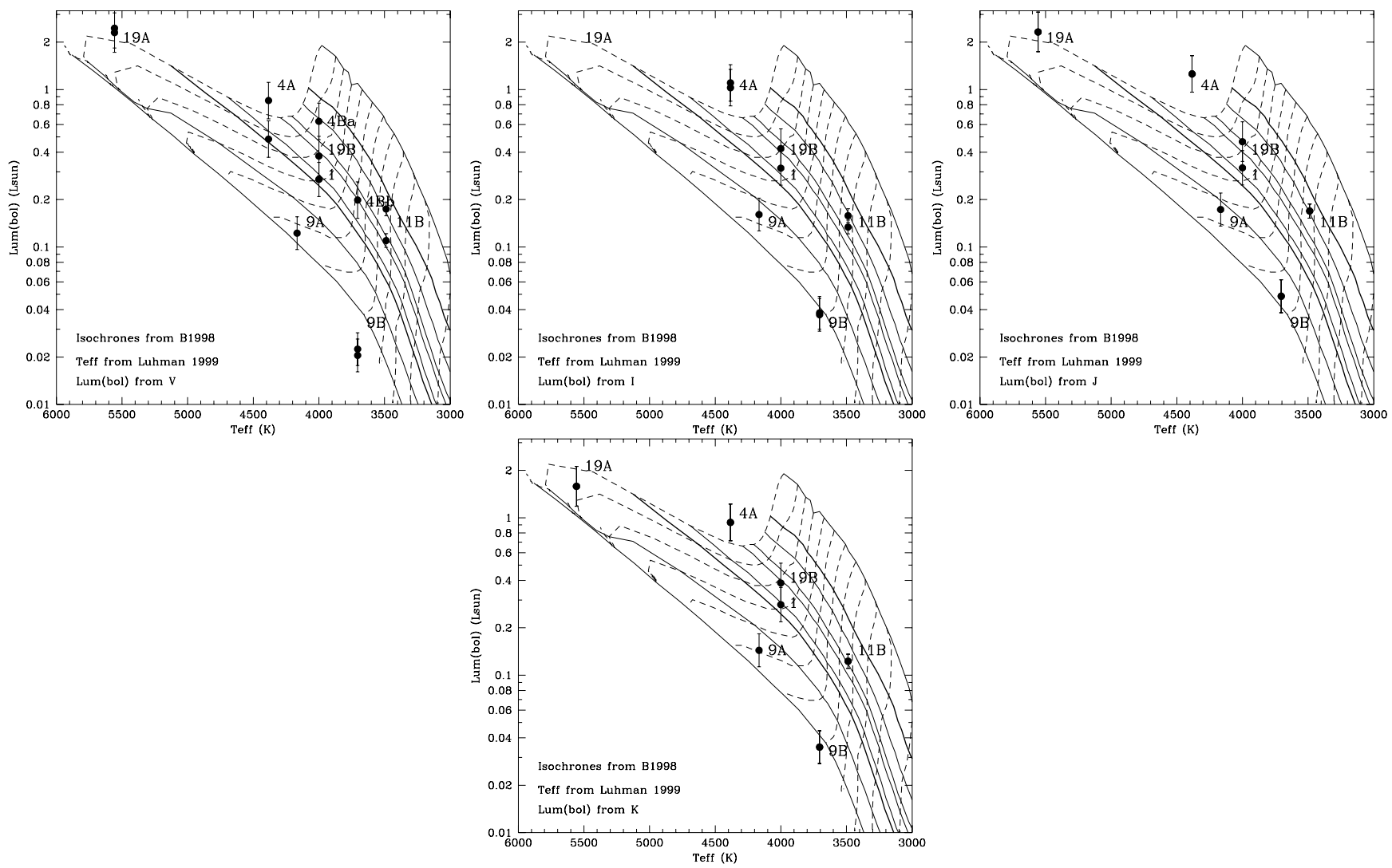

Fig. 7. Bolometric luminosity - in logarithmic scale - versus the effective temperature. The luminosities have been derived from the filters $V$, $I, J$ and $K$ and the corresponding bolometric corrections (see text) in different panels. The evolutionary isochrones - solid lines - and tracks dashed lines - correspond to models by Baraffe et al. (1998), whereas the effective temperatures were derived with the scale by Luhman (1999) for intermediate gravity. Errors in luminosities include the error in the distance and the photometric error when available. 
D. Barrado y Navascués: The age of TWA and 2M1207, Online Material $p 4$
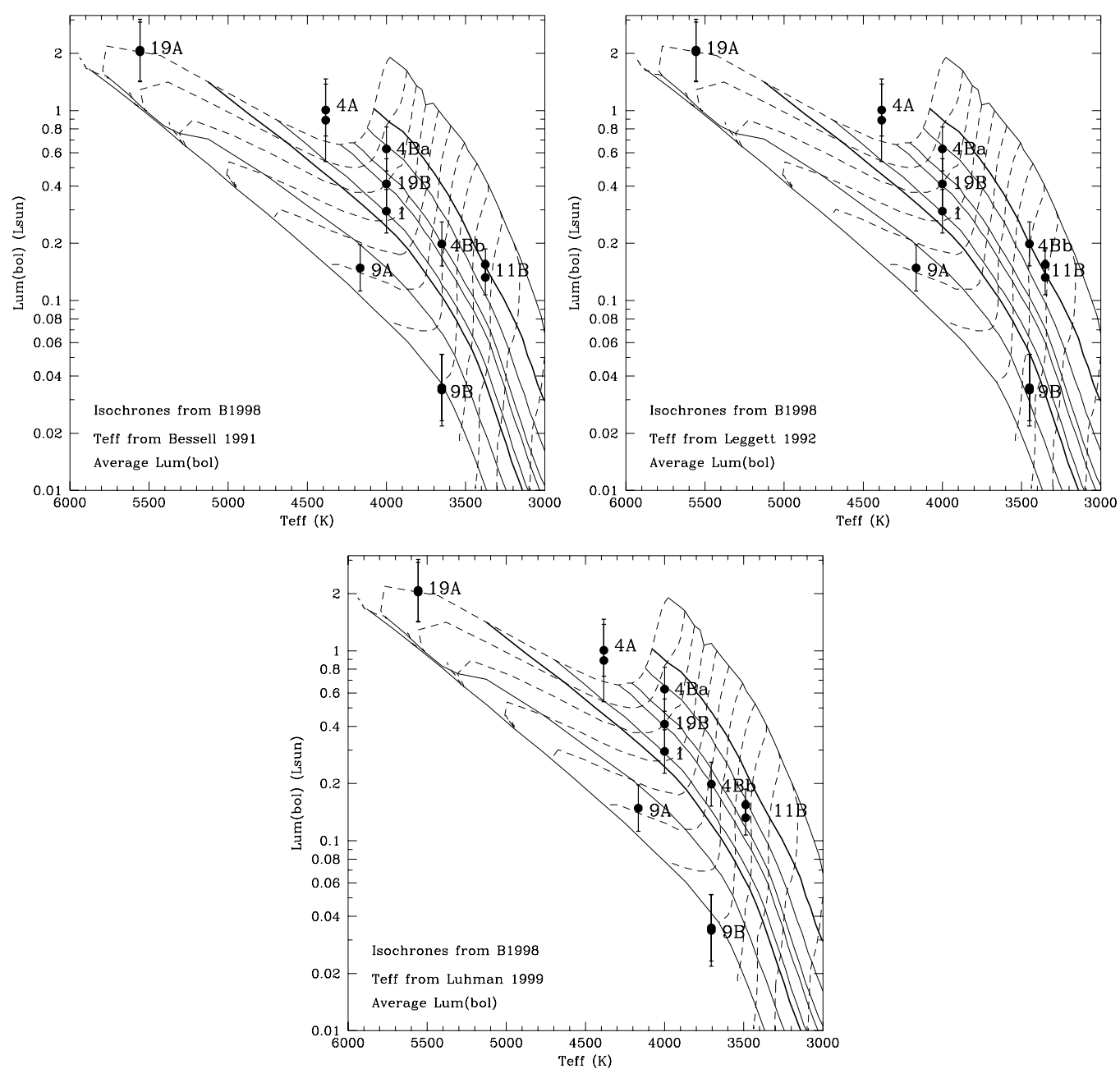

Fig. 8. Bolometric luminosity - in logarithmic scale - versus the effective temperature. The last values were computed using the temperature scales in the case of M spectral types of Bessell (1991), Leggett et al. (1992) and Luhman (1999). For warmer stars, Bessell (1979) was used in all three cases. The bolometric luminosities correspond to average values. Errors in luminosities include the error in the distance and the dispersion of the individual values used when computing the average. The evolutionary isochrones - solid lines - and tracks - dashed lines - correspond to models by Baraffe et al. (1998). 
D. Barrado y Navascués: The age of TWA and 2M1207, Online Material p 5
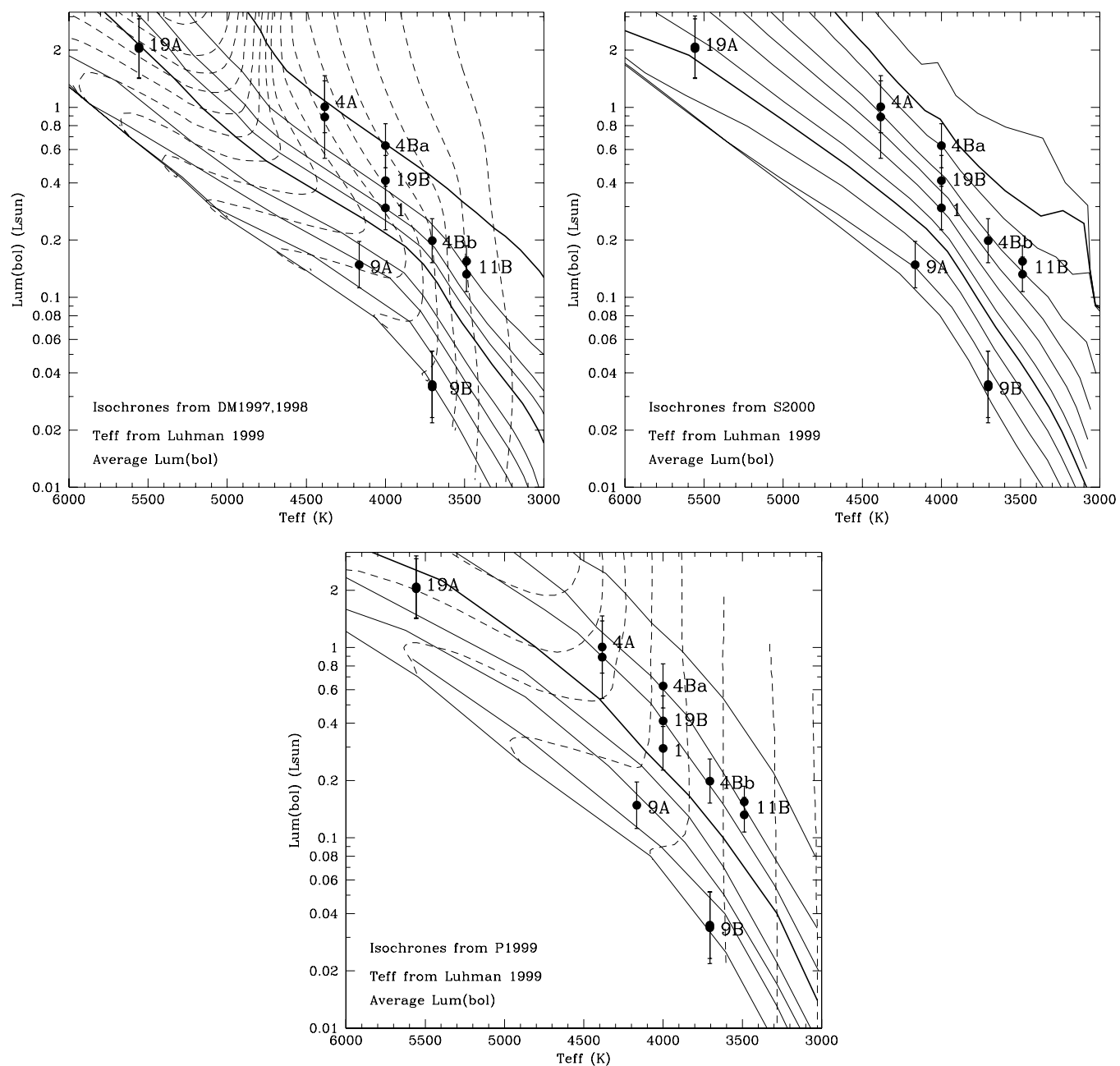

Fig. 9. Bolometric luminosity - in logarithmic scale - versus the effective temperature. The evolutionary isochrones and tracks correspond to models by D'Antona \& Mazzitelli (1997, 1998) Siess et al. (2000) and Palla \& Stahl (1999). Models by Baraffe et al. (1998) are displayed in the previous figure. The bolometric luminosities correspond to average values. Errors in luminosities include the error in the distance and the dispersion of the individual values used when computing the average. The effective temperatures were derived with the scale by Luhman (1999) for intermediate gravity. 
D. Barrado y Navascués: The age of TWA and 2M1207, Online Material p 6
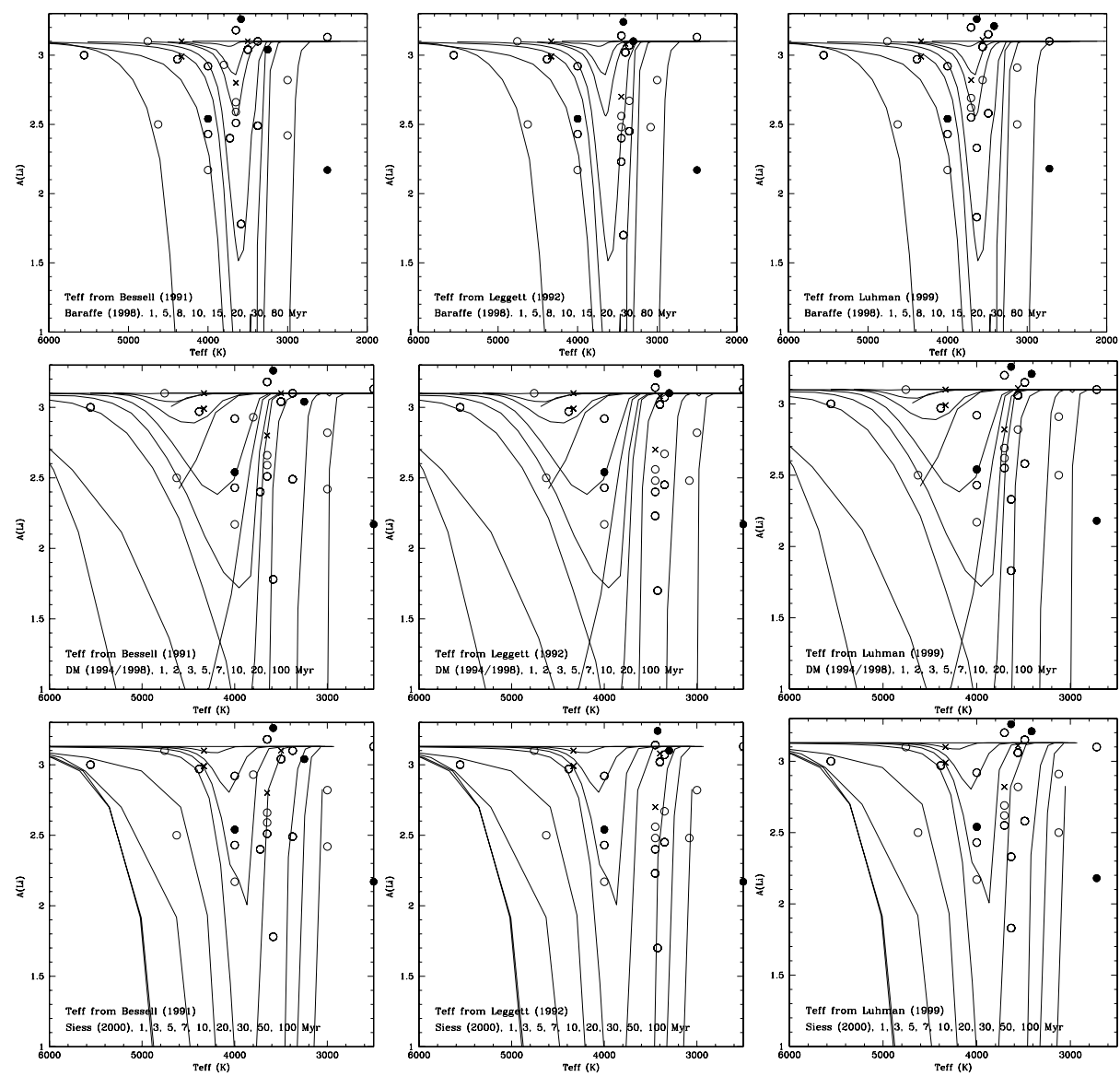

Fig. 13. Lithium abundance versus the spectral temperature for different models and temperature scales. TWA members are displayed as solid circles (CTT), open circles (WTT/PTT) and probable non-members (crosses). The 7/8 Myr Li depletion isochrone is highlighted in most of the figures. 
Table 1. Data for members of the TW Hydrae association.

\begin{tabular}{|c|c|c|c|c|c|c|c|c|c|c|}
\hline$\overline{\text { TWA }}$ & Sp. type & "ref. & $\overline{\bar{V}}$ & $\overline{\overline{I I}}$ & Parallax & $\overline{\bar{W} \text { (Halpha) }}$ & ref. & $\bar{~} \overline{W(\mathrm{Li})}$ & $\bar{~} \overline{\text { ref. }}$. & Name \\
\hline TWA-01 & K7e & $(02)$ & 10.92 & 9.18 & 17.72 & 220 & $(08)$ & 0.39 & $(08)$ & TWHya \\
\hline$"$ & K8Ve & (10) & - & - & - & 265 & (02) & 0.46 & (02) & - \\
\hline$"$ & - & - & - & - & - & 300. & (10) & 0.440 & (10) & - \\
\hline$"$ & - & - & - & - & - & 150.0 & (07) & 0.530 & (07) & - \\
\hline$"$ & - & - & - & - & - & 238.0 & (11) & 0.426 & (18) & - \\
\hline$"$ & - & - & - & - & - & 165.0 & (11) & 0.37 & (06) & - \\
\hline$"$ & - & - & - & - & - & 225 & (06) & - & - & - \\
\hline$"$ & - & - & - & - & - & 71.4-101 & (01) & - & - & - \\
\hline$"$ & - & - & - & - & - & 172.5 & (23) & - & - & - \\
\hline TWA-02A & M0.5 & (08) & 11.60 & 9.20 & - & 1.89 & (08) & 0.494 & (18) & CD-29d8887A \\
\hline$"$ & M2Ve & (10) & - & - & - & 2 & (02) & 0.49 & (08) & - \\
\hline$"$ & M0 & $(02)$ & - & - & - & 2.1 & (10) & 0.560 & (10) & - \\
\hline$"$ & - & - & - & - & - & - & - & 0.52 & (02) & - \\
\hline$"$ & - & - & - & - & - & 1.8 & (23) & - & - & - \\
\hline TWA-02B & M2 & (08) & 13.50 & 10.70 & - & 2.28 & (06) & 0.42 & (06) & CD-29d8887B \\
\hline TWA-03A & M3e & (02) & 12.00 & 9.60 & - & 21.8 & (08) & 0.53 & (08) & Hen3-600A \\
\hline$"$ & M3 & (08) & 12.04 & 9.10 & - & 10. & (10) & 0.610 & (10) & - \\
\hline$"$ & M4Ve & (10) & - & 9.08 & - & 20. & (02) & 0.55 & (02) & - \\
\hline$"$ & - & - & - & - & - & 26.0 & (11) & 0.563 & (18) & - \\
\hline$"$ & - & - & - & - & - & 27.0 & (11) & - & - & - \\
\hline$"$ & - & - & - & - & - & 22.6 & (23) & - & - & - \\
\hline TWA-03B & M3.5 & (08) & 13.70 & 8.86 & - & 7.14 & (08) & - & - & Hen3-600B \\
\hline$"$ & M3 & $(02)$ & - & 10.10 & - & 8. & (02) & 0.54 & (08) & - \\
\hline$"$ & - & - & - & - & - & 6.7 & (11) & 0.50 & (02) & - \\
\hline$"$ & - & - & - & - & - & 6.1 & (23) & - & - & - \\
\hline TWA-04A & K4-K5 & (05) & 8.89 & 7.50 & 21.43 & 0. & (14) & 0.360 & (14) & HD98800A \\
\hline$"$ & K7V & (10) & 9.41 & 7.39 & - & 0.0 & (11) & 0.425 & (05) & - \\
\hline$"$ & - & - & - & - & - & 0 . & (10) & 0.430 & (10) & - \\
\hline TWA-04Ba & K7 & (05) & 9.71 & - & 21.43 & - & - & 0.335 & (05) & HD98800Ba \\
\hline TWA-04Bb & M1 & (05) & 11.47 & - & 21.43 & - & - & 0.450 & (05) & HD98800Bb \\
\hline TWA-05Aab & M1.5 & (08) & 11.54 & 9.01 & - & 13.4 & (08) & 0.57 & (08) & CD-33d7795A \\
\hline$"$ & M3Ve & (10) & 11.72 & 9.10 & - & 10. & (10) & 0.540 & (10) & - \\
\hline$"$ & - & - & - & - & - & 7.2 & (15) & 0.6 & (15) & - \\
\hline$"$ & - & - & - & - & - & 6.6 & (15) & 0.572 & (18) & - \\
\hline$"$ & - & - & - & - & - & 11.5 & (23) & - & - & - \\
\hline TWA-05B & M8.5 & (08) & 20.40 & 15.8 & - & 5.1 & (15) & 0.3 & (15) & CD-33d7795B \\
\hline$"$ & - & - & - & 15.80 & - & 20. & (09) & - & - & - \\
\hline TWA-06 & K7 & (08) & 11.62 & 9.94 & - & 4.65 & (08) & 0.56 & (08) & TYC7183-1477-1 \\
\hline$"$ & - & - & 12.00 & 9.77 & - & 3.4 & (23) & - & - & - \\
\hline TWA-07 & M1 & (08) & 11.65 & 9.21 & - & 4.95 & (08) & 0.44 & (08) & TYC7190-2111-1 \\
\hline$"$ & - & - & 11.06 & 9.10 & - & 5.8 & (23) & - & - & - \\
\hline TWA-08A & M2 & (08) & 12.23 & 9.82 & - & 7.34 & (08) & 0.53 & (08) & RXJ1132.7-2651 \\
\hline$"$ & M2Ve & (10) & - & 9.61 & - & 25. & (10) & 0.520 & (10) & - \\
\hline$"$ & - & - & - & - & - & 8.0 & (23) & - & - & - \\
\hline TWA-08B & M5 & (08) & - & 11.48 & - & 16.5 & (08) & 0.56 & (08) & - \\
\hline$"$ & - & - & - & - & - & 13.3 & (23) & - & - & - \\
\hline TWA-09A* & K5 & (08) & 11.26 & 9.64 & 19.87 & 2.35 & (08) & 0.46 & (08) & CD-36d7429A \\
\hline$"$ & - & - & 11.32 & 9.60 & - & 3.2 & (11) & 0.47 & (18) & - \\
\hline$"$ & - & - & - & - & - & 2.1 & (23) & - & - & - \\
\hline TWA-09B* & M1 & (08) & 14.00 & 11.42 & 19.87 & 5.01 & (08) & 0.48 & (08) & - \\
\hline ” & - & - & 14.10 & 11.45 & - & 5.1 & (11) & - & - & - \\
\hline$"$ & - & - & - & - & - & 4.3 & (23) & - & - & - \\
\hline
\end{tabular}

${ }^{*}$ Member?

$(01)=$ Rucinski \& Krautter (1983); (02)= de la Reza et al. (1989); (03)= Jura et al. (1993); (04)= Stauffer et al. (1995); (05)= Soderblom et al. (1996); (06)= Hoff et al. (1998); (07)= Sterzik et al. (1999); (08)= Webb et al. (1999); (09)= Neuhauser et al. (2000); (10)= Torres et al. (2000); $(11)=$ Muzerolle, priv. comm (2000); (12)= Zuckerman et al. (2001); (13)= Gizis (2002); (14)= Song et al. (2002); (15)= Mohanty et al. (2003); $(16)=$ Reid et al. (2003); (17)= Song et al. (2003); (18)= Torres et al. (2003); (19)= Gizis et al. (2004); (20)= Weinberger et al. (2004); (21)= Zuckerman \& Song (2004); (22)= Scholz \& Jayawardhana (2006); (23)= Jayawardhana et al. (2006); (24)= Scholz et al. (2006); (24)= this paper. 
Table 1. continued.

\begin{tabular}{|c|c|c|c|c|c|c|c|c|c|c|}
\hline$\overline{\text { TWA }}$ & Sp. type & ref. & $\overline{\bar{V}}$ & $\bar{I}$ & Parallax & $\overline{W(\text { Halpha) }}$ & ref & $\overline{W(\mathrm{Li})}$ & ref. & Name \\
\hline TWA-10 & M2.5 & $(08)$ & 12.96 & 10.49 & - & 8.39 & $(08)$ & 0.46 & $(08)$ & 1RXSJ123504.4-413629 \\
\hline$"$ & - & - & 12.70 & 10.50 & - & 7.1 & (11) & - & - & - \\
\hline$"$ & - & - & - & - & - & 4.5 & (11) & - & - & - \\
\hline$"$ & - & - & - & - & - & 13.6 & (23) & - & - & - \\
\hline TWA-11A & A0 & (08) & 5.78 & 5.81 & 14.91 & - & - & - & - & HR4796A \\
\hline TWA-11B & M2.5 & (08) & 13.3 & 10.78 & 14.91 & 3.5 & (04) & 0.550 & (04) & HR4796B \\
\hline$"$ & - & - & 12.80 & 10.60 & - & 3.5 & (23) & - & - & - \\
\hline TWA-12 & M2 & (07) & 12.85 & 10.49 & - & 4.5 & (07) & 0.530 & (07) & RXJ1121.1-3845 \\
\hline$"$ & - & - & 13.60 & 11.35 & - & 5.3 & (11) & - & - & - \\
\hline$"$ & - & - & - & - & - & 4.8 & (23) & - & - & - \\
\hline TWA-13A & M2e & (07) & 11.46 & - & - & 4.0 & (07) & 0.650 & (07) & RXJ1121.3-3447N \\
\hline$"$ & - & - & 12.10 & 10.10 & - & 4.0 & (11) & 0.650 & (18) & - \\
\hline$"$ & - & - & - & - & - & 3.0 & (23) & - & - & - \\
\hline TWA-13B & M1e & (07) & 12.00 & - & - & 2.8 & (07) & 0.550 & (07) & RXJ1121.3-3447S \\
\hline$"$ & - & - & 12.40 & 10.10 & - & 1.8 & (11) & 0.570 & (18) & - \\
\hline$"$ & - & - & - & - & - & 3.0 & (23) & - & - & - \\
\hline TWA-14 & M0 & (12) & 13.80 & 10.95 & - & 8.2 & (12) & 0.600 & (12) & 1RXSJ111325.1-4523 44 \\
\hline$"$ & - & - & - & - & - & 12.5 & (11) & - & - & - \\
\hline$"$ & - & - & - & - & - & 10.7 & (23) & - & - & - \\
\hline TWA-15A & M1.5 & (12) & 14.10 & 11.94 & - & 9.0 & (12) & 0.650 & (12) & 1RXSJ123420.1-4815 14 \\
\hline$"$ & - & - & - & 11.94 & - & 9.2 & (11) & - & - & - \\
\hline$"$ & - & - & - & - & - & 8.8 & (23) & - & - & - \\
\hline TWA-15B & M2 & (12) & 14.00 & 11.94 & - & 10.5 & (12) & 0.540 & (12) & - \\
\hline$"$ & - & - & - & 11.81 & - & 8.6 & (23) & - & - & - \\
\hline TWA-16 & M1.5 & (12) & 12.30 & 10.17 & - & 4.0 & (12) & 0.360 & (12) & 1RXSJ123456.1-453808 \\
\hline$"$ & - & - & - & 10.17 & - & 3.6 & (11) & - & - & - \\
\hline$"$ & - & - & - & - & - & 4.0 & (23) & - & - & - \\
\hline TWA-17 & K5 & (12) & 12.70 & 10.78 & - & 2.5 & (12) & 0.490 & (12) & 1RXSJ132046.5-461139 \\
\hline$"$ & - & - & - & 10.78 & - & 2.7 & (11) & - & - & - \\
\hline$"$ & - & - & - & - & - & 3.2 & (23) & - & - & - \\
\hline TWA-18 & M0.5 & (12) & 12.90 & 10.92 & - & 3.5 & (12) & 0.420 & (12) & 1RXSJ132137.0-442133 \\
\hline$"$ & - & - & - & 10.92 & - & 3.4 & (11) & - & - & - \\
\hline$"$ & - & - & - & - & - & 3.3 & (23) & - & - & - \\
\hline TWA-19A* & G5 & (12) & 9.07 & - & 9.62 & -2.6 & (12) & 0.190 & (12) & HD102458 \\
\hline$"$ & - & - & 9.14 & - & - & -0.57 & (14) & 0.189 & (14) & - \\
\hline$"$ & - & - & 9.10 & 8.40 & - & - & - & - & - & - \\
\hline TWA-19B* & K7 & (12) & 11.90 & 10.21 & 9.62 & 2.8 & (12) & 0.400 & (12) & 1RXSJ114724.3-495250 \\
\hline & - & - & - & - & - & 2.2 & (23) & - & - & - \\
\hline TWA-21 & $\mathrm{K} 3 / 4$ & (21) & 9.8 & - & - & 0.0 & (17) & 0.290 & (17) & TYC8599-0697 \\
\hline TWA-22 & M5 & (21) & 14.0 & - & - & 8.3 & (17) & 0.510 & (17) & SSS101726.7-535428 \\
\hline$"$ & - & - & - & - & - & 11.5 & (23) & - & - & - \\
\hline TWA-23 & M1 & (20) & 12.67 & - & - & 2.2 & (17) & 0.460 & (17) & SSS120727.4-324700 \\
\hline " & - & - & - & - & - & 2.4 & (23) & - & - & - \\
\hline TWA-24 & K3 & (21) & 12.26 & - & - & 0.5 & (17) & 0.337 & (17) & TYC8644-0802 \\
\hline & - & - & - & - & - & 0.3 & (23) & - & - & - \\
\hline TWA-25 & M0 & (20) & 11.36 & - & - & 1.8 & (17) & 0.494 & (17) & TYC7760-0283 \\
\hline & - & - & - & - & - & 2.4 & (23) & - & - & - \\
\hline TWA-26 & M8 & (13) & 20.50 & 15.88 & - & 300. & (13) & 0.5 & (15) & 2MJ1207334-393254 \\
\hline$"$ & - & - & - & 16.00 & - & 42. & (19) & 0.4 & (24) & - \\
\hline$"$ & - & - & - & - & - & 27.7 & (15) & 0.51 & (25) & - \\
\hline$"$ & - & - & - & - & - & $12-387$ & (22) & - & - & - \\
\hline$"$ & - & - & - & - & - & 44.7 & (25) & - & - & - \\
\hline TWA-27 & M8 & (13) & 20.20 & 15.70 & - & 10. & (13) & 0.5 & (15) & 2MJ1139511-315921 \\
\hline$"$ & - & - & - & - & - & 7.3 & (15) & 0.62 & (25) & - \\
\hline$"$ & - & - & - & - & - & 10.2 & (25) & - & - & - \\
\hline
\end{tabular}

${ }^{*}$ Member?

$(01)=$ Rucinski \& Krautter (1983); (02)= de la Reza et al. (1989); (03)= Jura et al. (1993); (04)= Stauffer et al. (1995); (05)= Soderblom et al. (1996); (06)= Hoff et al. (1998); (07)= Sterzik et al. (1999); (08)= Webb et al. (1999); (09)= Neuhauser et al. (2000); (10)= Torres et al. (2000); $(11)=$ Muzerolle, priv. comm (2000); (12)= Zuckerman et al. (2001); (13)= Gizis (2002); (14)= Song et al. (2002); (15)= Mohanty et al. (2003); $(16)=$ Reid et al. (2003); (17)= Song et al. (2003); (18)= Torres et al. (2003); (19)= Gizis et al. (2004); (20)= Weinberger et al. (2004); (21)= Zuckerman \& Song (2004); (22)= Scholz \& Jayawardhana (2006); (23)= Jayawardhana et al. (2006); (24)= Scholz et al. (2006); (24)= this paper. 